\title{
Novel loss-of-function mutation in $B R C A 2$ gene identified in a Chinese female with a family history of ovarian cancer: A case report
}

\author{
YANZHI CUI $^{1 *}$, YANYAN WANG $^{2 *}$, NINGZHI ZHANG ${ }^{3 *}, \mathrm{JUN} \mathrm{HE}^{4 *}, \mathrm{HUI} \mathrm{HUANG}^{2}$, \\ FENGLING LIU ${ }^{1}$, SUJU WEI ${ }^{1}$, QIAN DONG ${ }^{1}$, JING WU ${ }^{2}$, KEKE LIN $^{2}$, WEIXI CHEN ${ }^{2}$, JIALE XIANG ${ }^{2}$, \\ HUI JIN ${ }^{1}$, ZHIYU PENG ${ }^{2}$, QIANG ZHAO ${ }^{5}$, WEI LI ${ }^{2}$, DA JIANG ${ }^{1}$ and SANTASREE BANERJEE ${ }^{2}$ \\ ${ }^{1}$ Department of Internal Medicine, The Fourth Hospital of Hebei Medical University, Shijiazhuang, Hebei 050000; \\ ${ }^{2}$ BGI Genomics, BGI-Shenzhen, Shenzhen, Guangdong 518083; ${ }^{3}$ Prenatal Diagnosis Center, Department of Obstetrics, \\ Fuyang People's Hospital, Fuyang, Anhui 236000; ${ }^{4}$ Changsha Hospital for Maternal and Child Health Care, \\ Changsha, Hunan 410007; ${ }^{5}$ Department of Obstetrics and Gynecology, Jiangmen Central Hospital, \\ Affiliated Jiangmen Hospital of Sun Yat-Sen University, Jiangmen, Guangdong 529030, P.R. China
}

Received November 16, 2017; Accepted November 8, 2018

DOI: $10.3892 / 01.2019 .9950$

\begin{abstract}
Inherited loss-of-function mutations in the tumor suppressor $B R C A 2$ gene are associated with a high risk of ovarian cancer in the Chinese population. The current case report discusses a novel heterozygous insertion in BRCA2 gene, c.3195_3196insA, in a 54-year-old Chinese female with hereditary ovarian cancer. This frameshift mutation generates a premature stop codon at amino acid 1,076, which leads to a truncated BRCA2 protein instead of a wild-type BRCA2 protein with 3,418 amino acids. According to the Breast Cancer Information Core database, this mutation has not been previously reported. However, germline mutations of BRCA2 are a more prevalent cause of ovarian cancer in Chinese females compared with females in Western populations. The present study expands the mutational spectra of $B R C A 2$ that is associated with ovarian cancer.
\end{abstract}

\section{Introduction}

Among females, ovarian cancer is a frequently diagnosed cancer type and a leading cause of mortality (1). In

Correspondence to: Dr Santasree Banerjee, BGI Genomics, BGI-Shenzhen, Building 7, BGI Park, 21 Hongan 3rd Street, Yantian, Shenzhen, Guangdong 518083, P.R. China

E-mail: santasree.banerjee@bgi.com

Dr Da Jiang, Department of Internal Medicine, The Fourth Hospital of Hebei Medical University, 12 Jiankang Road, Shijiazhuang, Hebei 050000, P.R. China

E-mail: jiangda139@163.com

*Contributed equally

Key words: novel heterozygous mutations, truncated BRCA2 protein, tumor suppressor genes, frameshift mutation, ovarian cancer
China, the incidence rate of ovarian cancer has increased exponentially over the last decade (2). Inherited germline mutations in $B R C A 2$ predisposes individuals to a high risk of ovarian cancer. Therefore, genetic screening of BRCA2 mutations may be a significant component of clinical practice for individuals with a family history of ovarian cancer. In females, BRCA2 mutations result in a lifetime risk of $11-18 \%$ of developing ovarian cancer $(3,4)$. Hereditary ovarian cancer is an autosomal dominant syndrome with incomplete penetrance caused by germline mutations of $B R C A 2$. Germline mutations in both BRCA1 and BRCA2 are major causes of hereditary ovarian cancer $(5,6)$. BRCAl and $B R C A 2$ serve key roles in the double-strand break repair system (7). Based on the Breast Cancer Information Core (BIC) database (http://research.nhgri.nih.gov/bic/), approximately 3,700 $B R C A 1 / B R C A 2$ germline mutations have been associated with ovarian cancer. The present study reports a novel heterozygous germline insertion mutation in the BRCA2 gene, c.3195_3196insA. This single nucleotide insertion causes a frameshift mutation, which results in the formation of a truncated BRCA2 protein with 1,076 amino acids instead of the wild-type BRCA2 protein with 3,418 amino acids.

\section{Case report}

The proband investigated in the current study is a 54-year-old Chinese female clinically diagnosed with ovarian cancer who belongs to a non-consanguineous Chinese family. The proband's elder sister passed away at the age of 46 years as a result of ovarian cancer. The age and cause of mortality for the proband's parents are unknown (Fig. 1). The proband had been diagnosed with stage III right ovarian cancer, along with liver metastasis. The top edge of the liver exhibited a nodule with mild leukoaraiosis. The magnetic resonance imaging (MRI) scan demonstrated no metastasis around the brain. 
The biopsy sections (thickness, 3-4 $\mu \mathrm{m}$ ) were paraffin embedded, fixed with $4 \%$ neutral buffer formaldehyde solution, at room temperature $\left(25^{\circ} \mathrm{C}\right)$ for $6-48 \mathrm{~h}$. Pathological biopsy was performed at the Fourth Hospital of Hebei Medical University (Shijiazhuang, China). Hematoxylin and eosin staining of tumor tissue was performed by optical microscopy (magnification, $\mathrm{x} 460$ ) and microscopy images were obtained (Fig. 2A-C). Paraffin-embedded slices were stained with IHC markers (Ventana Benchmark Ultra automatic immunohistochemical staining platform, primary antibodies: ER: Anti-estrogen receptor (SP1) rabbit monoclonal primary antibody, Roche, catalog number: 790-4325; PR: Anti-progesterone receptor (1E2) rabbit monoclonal primary antibody, Roche, catalog number: 790-4296; P53: Anti-P53 mouse monoclonal primary antibody, Roche, catalog number: 800-2912; Ki-67: Anti-Ki-67 (30-9) rabbit monoclonal primary antibody, Roche, catalog number: 790-4286; CEA: Anti-CEA (COL-1) mouse monoclonal primary antibody, From: MXB Biotechnologies, catalog number: Kit-0008; CA125: Anti-CA125 (TA347) mouse monoclonal primary antibody, MXB Biotechnologies, catalog number: MAB-0007; WT1: Anti-WT1 (MX012) mouse monoclonal primary antibody, MXB Biotechnologies, catalog number: MAB-0678; PAX-8: Anti-PAX-8 rabbit polyclonal primary antibody, MXB Biotechnologies, catalog number: RAB-0657; PAX-2: MXB Biotechnologies, catalog number: RAB-0648. Secondary antibodies: MaxVision DAB, From: MXB Biotechnologies, catalog number: KIT-5220. All antibodies were purchased in their ready-to-use format so dilutions weren't required. IHC experimental procedure according to the manufacturer's protocols), the percentage of positive staining was calculated by eye. Immunohistochemistry (IHC) for estrogen receptors demonstrated a high (70\%) expression level in cancer cells (Fig. 2D). IHC for progesterone receptors revealed a moderate $(30 \%)$ expression level in cancer cells (Fig. 2E). IHC for p53 demonstrated zero expression level of p53 in cancer cells (Fig. 2F). IHC for Ki67 revealed a moderate $(30 \%)$ expression level in cancer cells (Fig. 2G). Staining for cancer antigen 125 demonstrated a high expression level (Fig. 2H). IHC for Wilms tumor protein (WT1) revealed a moderate expression level in cancer cells (Fig. 2I). IHC for paired box gene PAX-2, PAX-8 and CEA was also performed, revealing zero, positive and positive expression, respectively (Fig. 2J-L). An MRI scan was performed, which demonstrated enhancement of the pelvic tumor (Fig. 3A), enhancement of hepatic metastasis (Fig. 3B,C), and thickening of the omentum majus and tumor tissue (Fig. 3D).

The patient's treatment regimen consisted of TC scheme (paclitaxel and carboplatin: Paclitaxel liposome, $240 \mathrm{mg} / \mathrm{day}$ $1+$ carboplatin, $0.5 \mathrm{~g} / \mathrm{day} 1$, intravenous injection) chemotherapy for two cycles. Following treatment, the patient showed an improvement, and a gynecological surgeon contacted the patient's family.

Prior to the experiment, written informed consent was obtained from each patient. Peripheral blood samples of the patients were collected and genomic DNA was extracted. Targeted high-throughput sequencing (DNA polymerase is T4 DNA POLYMERASE, supplier is ENZYMATICS; Qiagen, Inc., Valencia, CA, USA) was performed with a panel of a 21 genes (BRCA1, BRCA2, CHEK2, PALB2, BRIP1, TP53, PTEN, STK11, CDH1, ATM, BARD1, MLH1, MRE11A, MSH2,

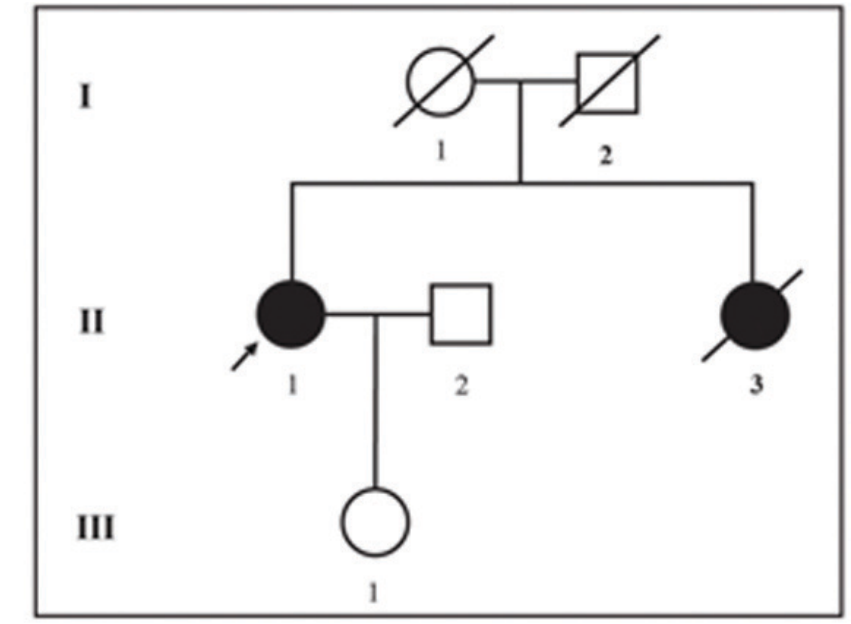

Figure 1. Pedigree structure of the family of the Chinese patient with hereditary breast cancer. Black shapes indicate individuals with hereditary ovarian cancer. Squares and circles denote males and females, respectively. Individuals labeled with a diagonal line are deceased. Roman numerals indicate generations. The arrow indicates the proband.

MSH6, MUTYH, NBN, PMS1, PMS2, RAD50 and RAD51C). Roche NimbleGen's (Roche Applied Science, Madison, MI, USA) custom Sequence Capture Human array was used to designed to capture targeted sequence, according to the manufacturer's protocols. A target area of $172,959 \mathrm{bp}$, a target area coverage of $99.49 \%$ and a target area average depth of 586.13x. Samples were sequenced simultaneously on Illumina HiSeq 2500 Analyzers (Illumina Inc., San Diego, CA, USA) for 90 cycles according to the manufacturer's protocols. Targeted high-throughput sequencing identified a novel heterozygous mutation, c.3195_3196insA, in BRCA2, which causes a frameshift mutation that generates a premature stop codon and results in the formation of a truncated BRCA2 protein. This mutation was confirmed by Sanger sequencing (Fig. 1R). The mutation was absent in the Human Gene Mutation database (www.hgmd.cf.ac.uk/) and the Online Mendelian Inheritance in Man database (www.omim.org). In addition, the mutation could not be identified in our in-house databases, which contains $\sim 30,000$ samples from Chinese Han individuals.

Since the proband's elder sister passed away at an age of 46 years due to ovarian cancer, it can be hypothesized that she carried the same mutation in BRCA2. In addition, the proband's father or mother may carry the same mutation. However, as the proband's elder sister and parents had passed away, it was not possible to investigate this. The proband's children did not volunteer genetic testing. Therefore, only the following suggestions can be made. Firstly, it could be hypothesized that the proband and her sister carried the same mutation on the $B R C A 2$ gene, which was inherited from their father or mother. By contrast, it could be hypothesized that the mutation carried by the proband is a de novo mutation. In this case, her parents and sister would not have carried the same mutation. With the knowledge that the proband's sister passed away due to ovarian cancer, we speculate that the first hypothesis is more likely. The Ethical Committee of The Fourth Hospital of Hebei Medical University (Shijiazhuang, China) approved the current study protocol, which was in compliance with the Declaration of Helsinki. 
A

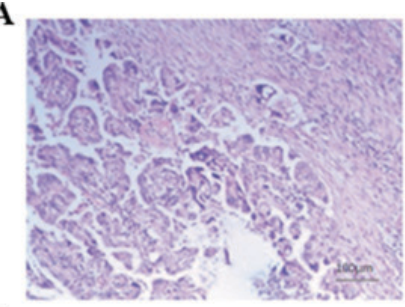

D
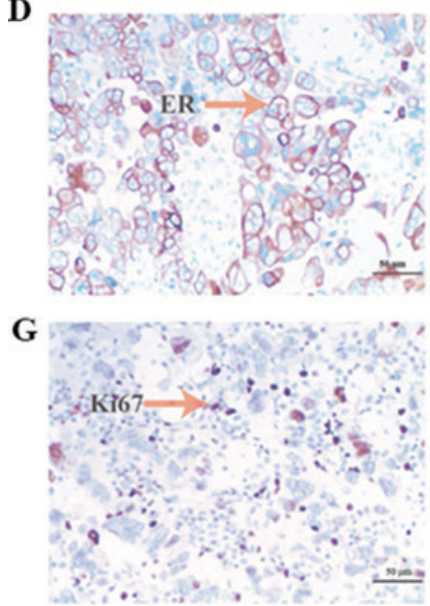

$\mathbf{J}$

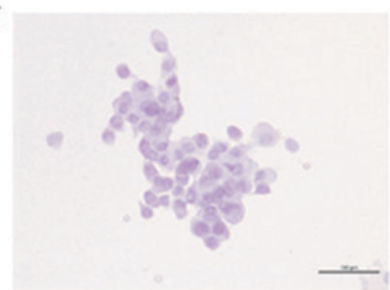

B

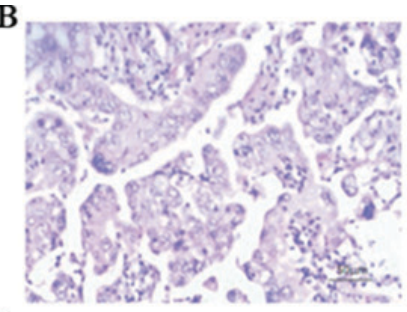

E

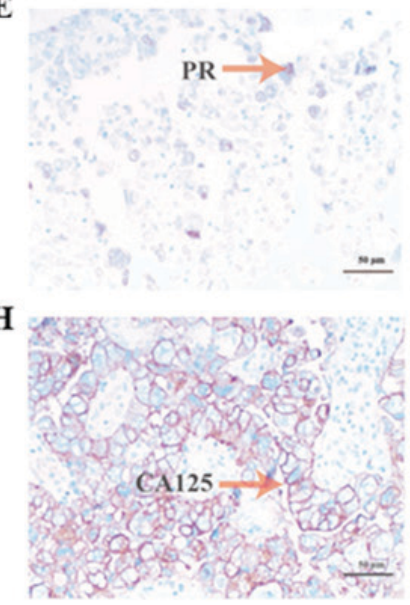

$\mathbf{K}$

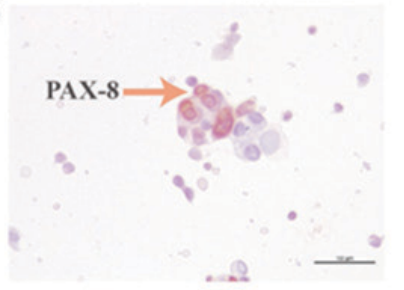

C

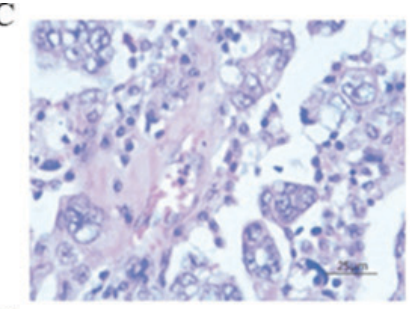

$\mathbf{F}$

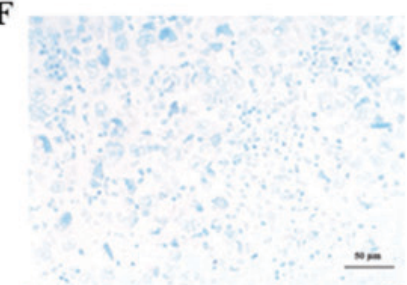

I

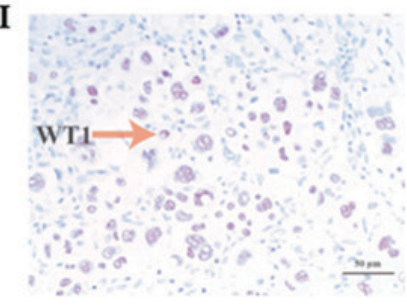

$\mathbf{L}$

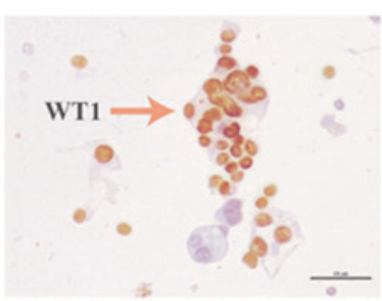

Figure 2. Hematoxylin and eosin staining of tumor tissue was performed, and images were obtained with a (A) scale bar=100 $\mu \mathrm{m}$, (B) scale bar=50 $\mu \mathrm{m}$ and (C) scale bar $=25 \mu \mathrm{m}$. (D) Immunohistochemistry for ER (red arrow) indicated a high (70\%) expression level in cancer cells. Scale bar=50 $\mu \mathrm{m}$. (E) Immunohistochemistry for PR (red arrow) demonstrated a moderate (30\%) expression level in cancer cells. Scale bar=50 $\mu \mathrm{m}$. (F) Immunohistochemistry for p53 indicated no expression in cancer cells. Scale bar=50 $\mu \mathrm{m}$. (G) Immunohistochemistry for Ki67 (red arrow) revealed a moderate (30\%) expression level in cancer cells. Scale bar=50 $\mu \mathrm{m}$. (H) Immunohistochemistry for CA125 (red arrow) demonstrated a high expression level in cancer cells. Scale bar=50 $\mu \mathrm{m}$. (I) Immunohistochemistry for WT1 (red arrow) indicated a moderate expression level in cancer cells. Scale bar $=50 \mu \mathrm{m}$. Positive expression was represented by a brownish color in the cytoplasm and membrane. (J) Immunocytochemistry for PAX-2. Scale bar=100 $\mu \mathrm{m}$. (K) Immunocytochemistry for PAX-8 (red arrow). Scale bar $=100 \mu \mathrm{m}$. (L) Immunocytochemistry for CEA (red arrow). Magnification, x460. ER, estrogen receptor; PR, progesterone receptor; CA125, cancer antigen 125; WT1, Wilms tumor protein; MRI, magnetic resonance imaging; PAX, paired box gene.

$\mathbf{A}$

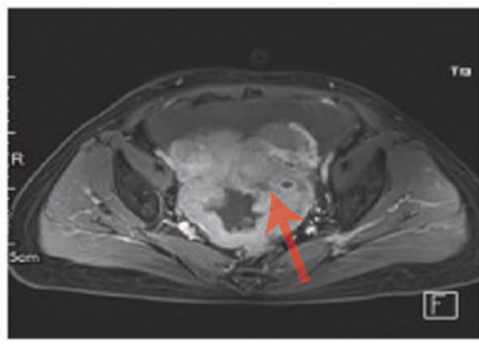

C

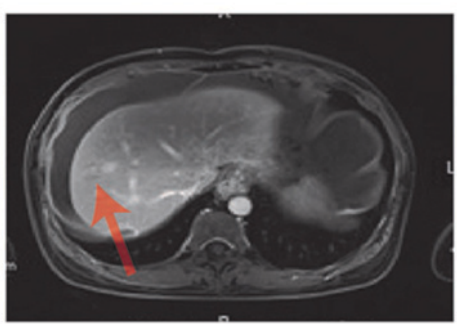

B

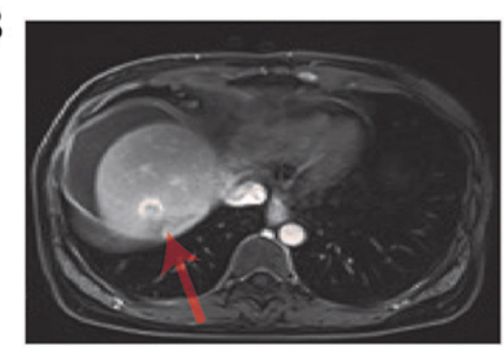

D

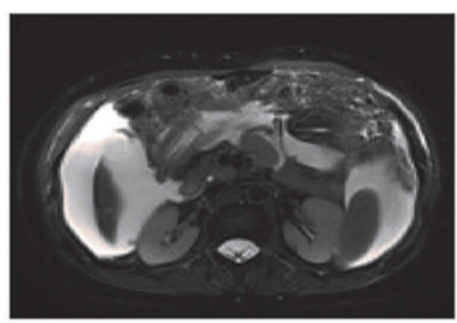

Figure 3. MRI of patient. (A) An MRI scan demonstrated enhancement of the pelvic tumor (red arrow). (B) An MRI scan indicated enhancement of hepatic metastasis (red arrow). (C) An MRI scan revealed enhancement of hepatic metastasis (red arrow). (D) An MRI scan identified thickening of the omentum majus and tumor tissue. 


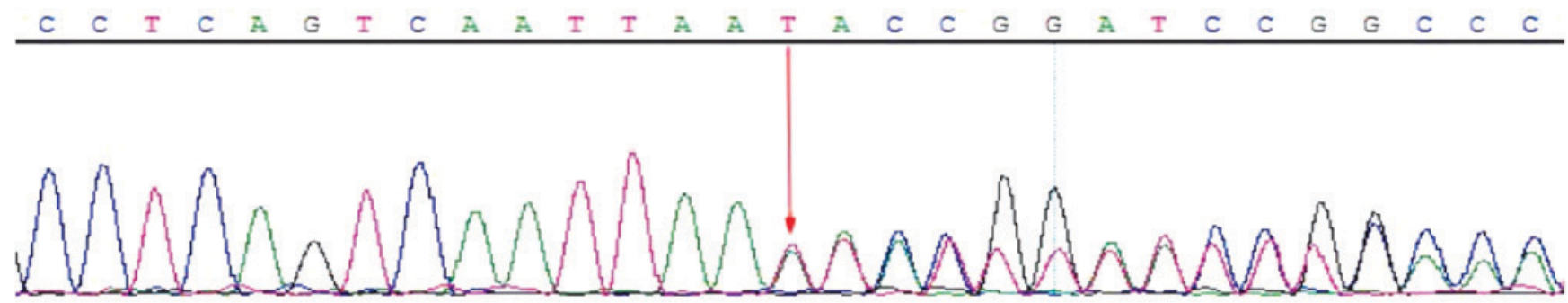

Figure 4. Sanger sequencing of the BRCA2 gene identified a germline novel heterozygous insertion mutation. The arrow indicates the novel heterozygous insertion mutation (c.3195_3196insA).

\section{Discussion}

A c.3195_3196insA frameshift mutation in exon 10 of the $B R C A 2$ gene, which has not previously been reported in the BIC database, was detected in a 54 year-old female Chinese patient diagnosed with ovarian cancer. Not all genetic variants are harmful; BRCA1/2 variations can be classified into the five following categories: 'Pathogenic', 'likely pathogenic', 'uncertain significance', 'likely benign' and 'benign', according to American College of Medical Genetics' guidelines (8). 'Pathogenic' or 'likely pathogenic' indicates that a mutation was identified in a specific gene, which increases the lifetime risk of developing certain cancer types, but does not indicate that cancer will definitely develop. Germline BRCA1/2 deleterious mutations lead to an increased risk of early onset hereditary breast and ovarian cancer (HBOC)-associated tumors, including breast, ovarian and pancreatic cancer in females, and to a lesser extent, breast and prostate cancer in males $(9,10)$.

Genetic sequencing techniques have developed radically during the past few decades; therefore, novel testing techniques have been widely used in the medical sector (11-15). For example, the enrichment step, a preparation technique used prior to next-generation sequencing (NGS), can significantly improve the accuracy and efficiency of medical screening (16). This technique was used in the current study to identify the largest possible number of mutations that may be associated with HBOC. Due to technology limitations, there are still many challenges in detecting copy-number variants by NGS (17), certain patients with a negative NGS testing result may also require multiplex ligation-dependent probe application, particularly patients with a family history of cancer.

$B R C A 1$ and BRCA2 were not the only genes analyzed in the current study; multiple gene analysis was performed. Numerous genes should be involved in sequencing tests as the mutational spectra of these genes can be accurately detected with NGS technology, which provides additional data that can support clinical diagnoses. However, involving multiple genes in sequencing tests may lead to the detection of a higher number of variants of uncertain significance (VUS) (18). Clinical recommendations may not be standardized for the presence of VUS mutations in certain cancer susceptibility genes, which may complicate results. These complex results are why suggestions from medical geneticists or certified genetic counselors are important. Since a number of NGS-based techniques are used in genetic testing and different genetic companies perform different techniques, customers should have access to the sensitivity and specificity of any results, as well as comparisons of various techniques (19). In our opinion, positive results should be validated using standard Sanger sequencing, which should also be available to relatives who are concerned about the risk of cancer.

\section{Acknowledgements}

Not applicable.

\section{Funding}

The current study was supported by the Special Foundation for High-level Talents of Guangdong (grant no. 2016TX03R171).

\section{Availability of data and materials}

The datasets used and/or analyzed in the present study are available from the corresponding author upon reasonable request.

\section{Authors' contributions}

SB, DJ, ZP designed and coordinated the study. DJ, YC, NZ, JH, FL, SW, QD, QZ and HJ assessed the clinical findings of the cases. YW, HH, JW, KL, WC, WL and JX performed the molecular genetic studies and analyzed the data. YW wrote the draft of the manuscript with input from the other co-authors. All authors read and approved the final manuscript.

\section{Ethics approval and consent to participate}

All study participants provided written informed consent and the study design was approved by an Institutional Ethics Review Board of the Department of Internal Medicine, The Fourth Hospital of Hebei Medical University (Shijiazhuang, China).

\section{Patient consent for publication}

A written consent was obtained from the patient for the publications of the present study.

\section{Competing interests}

The authors declare that they have no competing interests. 


\section{References}

1. Torre LA, Bray F, Siegel RL, Ferlay J, Lortet-Tielent J and Jemal A: Global cancer statistics, 2012. CA Cancer J Clin 65: 87-108, 2015

2. Chen W, Zheng R, Baade PD, Zhang S, Zeng H, Bray F, Jemal A, Yu XQ and He J: Cancer statistics in China, 2015. CA Cancer J Clin 66: 115-132, 2016.

3. Chen S and Parmigiani G: Meta-analysis of BRCA1 and BRCA2 penetrance. J Clin Oncol 25: 1329-1333, 2007.

4. Mavaddat N, Peock S, Frost D, Ellis S, Platte R, Fineberg E, Evans DG, Izatt L, Eeles RA, Adlard J, et al: Cancer risks for BRCA1 and BRCA2 mutation carriers: Results from prospective analysis of EMBRACE. J Natl Cancer Inst 105: 812-822, 2013.

5. Claus EB, Risch N and Thompson WD: Autosomal dominant inheritance of early $\square$ onset breast cancer. Implications for risk prediction. Cancer 73: 643-651, 1994.

6. Petrucelli N, Daly MB and Pal T: BRCA1-and BRCA2-associated hereditary breast and ovarian cancer: Adam MP, Ardinger HH, Pagon RA, Wallace SE, Bean LJH, Stephens K and Amemiya A (eds). University of Washington, Seattle, 2016.

7. Narod SA and Foulkes WD: BRCA1 and BRCA2: 1994 and beyond. Nat Rev Cancer 4: 665-676. 2004.

8. Richards S, Aziz N, Bale S, Bick D, Das S, Gastier-Foster J, Grody WW, Hegde M, Lyon E, Spector E, et al: Standards and guidelines for the interpretation of sequence variants: A joint consensus recommendation of the American college of medical genetics and genomics and the association for molecular pathology. Genet Med 17: 405-424, 2015.

9. Song H, Cicek MS, Dicks E, Harrington P, Ramus SJ, Cunningham JM, Fridley BL, Tyrer JP, Alsop J, et al: The contribution of deleterious germline mutations in BRCA1, BRCA2 and the mismatch repair genes to ovarian cancer in the population. Hum Mol Genet 23: 4703-4709, 2014.

10. Kim YC, Zhao L, Zhang H, Huang Y, Cui J, Xiao F, Downs B and Wang SM: Prevalence and spectrum of BRCA germline variants in mainland Chinese familial breast and ovarian cancer patients. Oncotarget 7: 9600-9612, 2016.

11. Lifton RP: Individual genomes on the horizon. N Engl J Med 362: 1235-1236, 2010.
12. Goossens D, Moens LN, Nelis E, Lenaerts AS, Glassee W, Kalbe A, Frey B, Kopal G, De Jonghe P, De Rijk P and Del-Favero J: Simultaneous mutation and copy number variation (CNV) detection by multiplex PCR-based GS-FLX sequencing. Hum Mutat 30: 472-476, 2009.

13. Daiger SP, Sullivan LS, Bowne SJ, Birch DG, Heckenlively JR, Pierce EA and Weinstock GM: Targeted high-throughput DNA sequencing for gene discovery in retinitis pigmentosa. Adv Exp Med Biol 664: 325-331, 2010.

14. Hoischen A, Gilissen C, Arts P, Wieskamp N, van der Vliet W, Vermeer S, Steehouwer M, de Vries P, Meijer R, Seiqueros J, et al: Massively parallel sequencing of ataxia genes after array-based enrichment. Hum Mutat 31: 494-499, 2010.

15. Chou LS, Liu CS, Boese B, Zhang X and Mao R: DNA sequence capture and enrichment by microarray followed by next-generation sequencing for targeted resequencing: Neurofibromatosis type 1 gene as a model. Clin Chem 56: 62-72, 2010.

16. Mamanova L, Coffey AJ, Scott CE, Kozarewa I, Turner EH, Kumar A,HowardE, Shendure J and Turner DJ: Target-enrichment strategies for next-generation sequencing. Nat Methods 7: 111-118, 2010.

17. Teo SM, Pawitan Y, Ku CS, Chia KS and Salim A: Statistical challenges associated with detecting copy number variations with next-generation sequencing. Bioinformatics 28: 2711-2718, 2012.

18. Kapoor NS, Curcio LD, Blakemore CA, Bremner AK, McFarland RE, West JG and Banks KC: Multigene panel testing detects equal rates of pathogenic BRCA $1 / 2$ mutations and has a higher diagnostic yield compared to limited BRCA1/2 analysis alone in patients at risk for hereditary breast cancer. Ann Surg Oncol 22: 3282-3288, 2015.

19. Mattocks CJ, Morris MA, Matthijs G, Swinnen E, Corveleyn A, Dequeker E, Müller CR, Pratt V and Wallace A; EuroGentest Validation Group: A standardized framework for the validation and verification of clinical molecular genetic tests. Eur J Hum Genet 18: 1276-1288, 2010. 